\title{
Arc Behaviour and Cathode Melting Process during VAR: an Experimental and Numerical Study
}

\author{
Alain JARDY, ${ }^{*}$ Pierre CHAPELLE, Ashish MALIK, Jean-Pierre BELLOT, Hervé COMBEAU \\ and Bernard DUSSOUBS
}

Institut Jean Lamour - UMR 7198 CNRS/Université de Lorraine, Département SI2M Ecole des Mines, Parc de Saurupt, CS 14243, F-54042 Nancy Cedex, France.

(Received on February 15, 2012; accepted on November 20, 2012)

\begin{abstract}
The present study aims to understand the melting of the consumable electrode in the VAR process and gain some insight into the influence of an ensemble arc motion on the melting behaviour. In a previous study, a 2D axisymmetric model of the heat transfer in the cathode had been developed. Using the operating parameters as model inputs, it enabled prediction of the melt rate and the evolution of the melting area. Model results were successfully compared to melt rate measurements in an industrial VAR furnace. In recent years, it has been claimed that the electric arc may not be considered as steady and axisymmetric. Our experimental investigation of the luminosity recorded during an actual VAR heat confirms that a transient 3D behaviour may take place. Therefore, a 3D version of the previous model was set up to predict the heat transfer and melting of the electrode, using the unknown ensemble arc motion as an input. The arc is assimilated to a transient distribution of energy flux density. Results evidence that the influence of the arc motion on the shape of the electrode tip can be very important. In industrial practice, the cathode tip usually remains relatively flat during melting. The shapes of the computed electrode tips enable us to propose some arc parameters which remain compatible with both the periodic behaviour of the light emitted and the flatness of the electrode.
\end{abstract}

KEY WORDS: Vacuum Arc Remelting; cathode melting; ensemble arc motion; numerical simulation.

\section{Introduction}

The principle of the Vacuum Arc Remelting (VAR) process, as illustrated in Fig. 1, consists in melting a consumable metallic electrode of the required grade under a high vacuum, in order to obtain a sound ingot of good structural quality. During remelting, an electric arc is maintained between the tip of the consumable electrode (usually the cathode) and the top of the secondary ingot (usually the anode), in order to ensure melting of the electrode. The metal droplets fall through the arc plasma and progressively build up the ingot, which solidifies in contact with the walls of a water-cooled copper crucible. This operation ensures both the purification of the metal, as it is gradually melted, and controlled solidification of the ingot in terms of structure and chemical homogeneity. In order to stabilize the arc, it can be confined with the aid of a magnetic field created by an external induction coil. This field can also be used to stir the liquid metal, the rotation induced being in the azimuthal direction. By reversing periodically the coil current, stirring can be alternated.

Vacuum Arc Remelting is currently used to produce high quality special steels and nickel-based superalloys. ${ }^{1,2)}$ It also represents the final stage in the melting cycle of reactive

* Corresponding author: E-mail: Alain.Jardy@ijl.nancy-universite.fr DOI: http://dx.doi.org/10.2355/isijinternational.53.213 metals, such as zirconium and titanium. ${ }^{3)}$ The strategic importance of these products and their very high added value make it essential to acquire a detailed understanding of the melting process. The work presented here is part of a

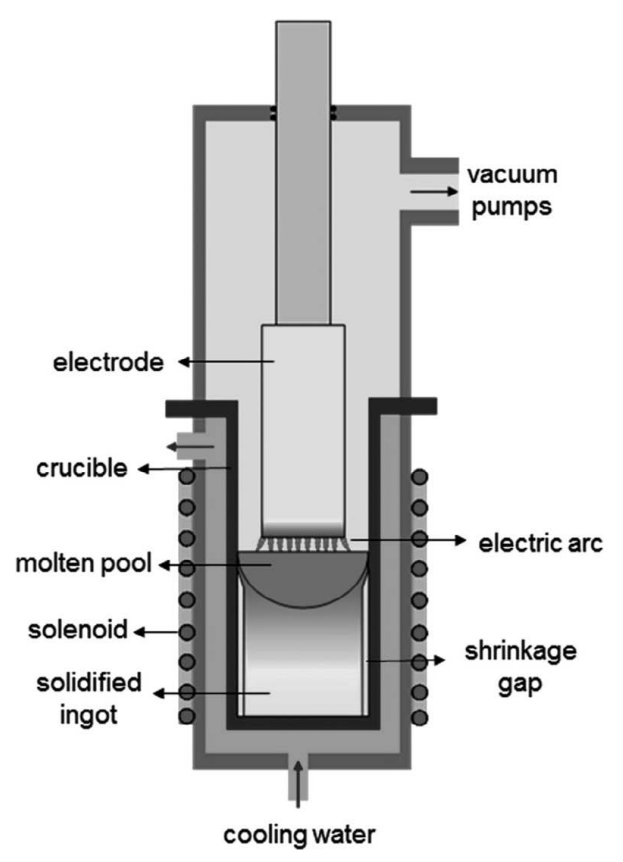

Fig. 1. A schematic representation of the VAR process. 
program initiated twenty years ago at Institut Jean Lamour (Nancy School of Mines) within the frame of industrial collaborations with several process users. ${ }^{4-6)}$ The aim is to develop a simulation software of the whole VAR operation, and subsequently to help optimizing the process.

In this paper, we focus on the macroscopic behaviour of the arc and the associated cathode melting process. Indeed, a thorough understanding of the dynamics of the arc is important for a proper control of the process performance and ingot quality. The spatial and temporal distribution of the arc determines the energy and electric current inputs to the ingot top surface and electrode tip, which control to a great extent the ingot and electrode evolution.

Direct knowledge of the electric arc behaviour in the VAR process is based on visualization studies performed first at Sandia National Laboratories ${ }^{7,8)}$ during the remelting of steel or Ni-based superalloy electrodes. Similar experiments have been carried out more recently by Chapelle et al. on zirconium electrodes. ${ }^{9}{ }^{10)}$ One of the main conclusions from these observations is that the behaviour of the arc is similar to the diffuse mode of a vacuum arc created between cold solid electrodes for the same range of current densities. The arc consists of several dispersed clusters of cathode spots moving very rapidly over the whole surface of the cathode, as shown on Fig. 2. Each cluster of spots is the source of a plasma jet expanding under vacuum in the electrode gap. No luminous arc column is observed in the gap.

Such a behaviour seems to imply that the total energy transferred from the arc to the cathode tip is distributed more or less uniformly; in particular, no azimuthal direction is privileged, so a $2 \mathrm{D}$ axisymmetric behaviour is expected at the macroscopic scale. This is consistent with the fact that the cathode tip remains relatively flat during full-scale melting. Figure 3 represents schematically the cathode behaviour in such a situation.

However, it has been recently reported that the arc often does not behave axisymmetrically at the macroscopic scale. Based on measurements of the luminosity and magnetic field created by the arc, ${ }^{11,12)}$ Ward et al. suggested that most of the time, the centre of gravity of the arc was off-centered and rotated in a time-averaged sense around the electrode axis with a constant speed (period equals typically 20 to 40 $\mathrm{s}$ when a superalloy electrode melts under nominally diffuse conditions). Then it was assumed that the distribution of current flow and heat input follows the distribution of this location and a part of the arc was assimilated to a loosely focused rotating spot, radially located away from the ingot centreline. Such a behaviour might result (see Fig. 4) in a 3D shape of the cathode tip. A 3D model of the ingot pool, using this representation as a boundary condition, ${ }^{13,14)}$ enabled several authors to conclude that the hydrodynamic behaviour of the melt pool and ingot solidification process can be greatly influenced by such an ensemble arc macroscopic motion.

However, the observation of an actual cathode tip, sometimes possible after a melt interruption, usually does not show the presence of any localized excavation corresponding to the rotation of a spot. This apparent contradiction requires more research study to be achieved. From the experimental point of view, new measurements are needed to characterize the arc behaviour. In parallel, it was decided

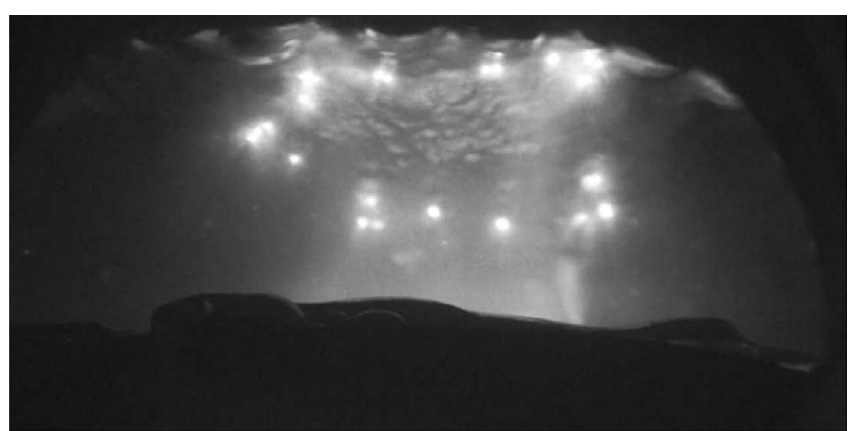

Fig. 2. Example of a video image of an electric arc during vacuum arc remelting of a zirconium alloy, showing clusters of cathode spots.

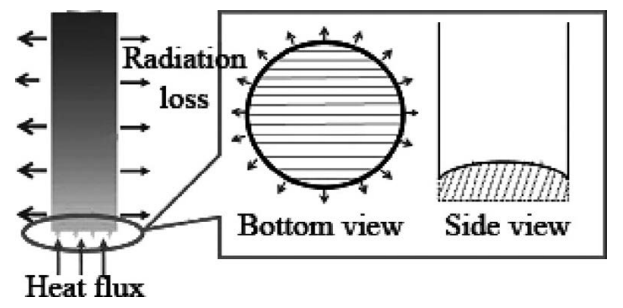

Fig. 3. Schematics of the heat flux density distribution at the cathode base (uniform heating $=2 \mathrm{D}$ assumption).

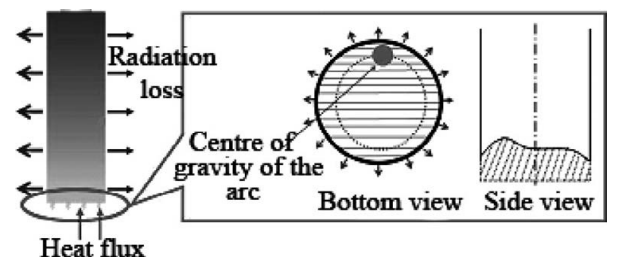

Fig. 4. Schematics of the heat flux density distribution at the cathode base (uniform heating + rotating 'arc-spot').

to simulate the cathode tip melting process in order to check the compatibility between the tip flatness and the existence of an ensemble arc motion.

\section{Observation of a Macroscopic Ensemble Motion of the Electric Arc}

In this section, we report a visual investigation of the dynamic behaviour of the electric arc in an industrial VAR furnace of coaxial type under various operating conditions. For this investigation, two synchronized video cameras positioned in front of two diametrically opposite viewing glasses on top of the furnace chamber were used to film the annulus gap between the crucible wall and the electrode (Fig. 5). This apparatus enabled us to observe only a portion of the ingot top surface area adjacent to the crucible wall and a luminous halo above it, which is generated by the arc. The arc itself, which is essentially established at the electrode bottom surface, was not directly visible to the cameras.

Video images were recorded during the melt of a zirconium alloy (Zy2) ingot. During this melt, five stirring sequences, which are listed in Table 1, were used successively in order to study the influence on the arc behaviour of the presence of an external axial magnetic field (as stated in the introduction of this paper, such a field is employed 


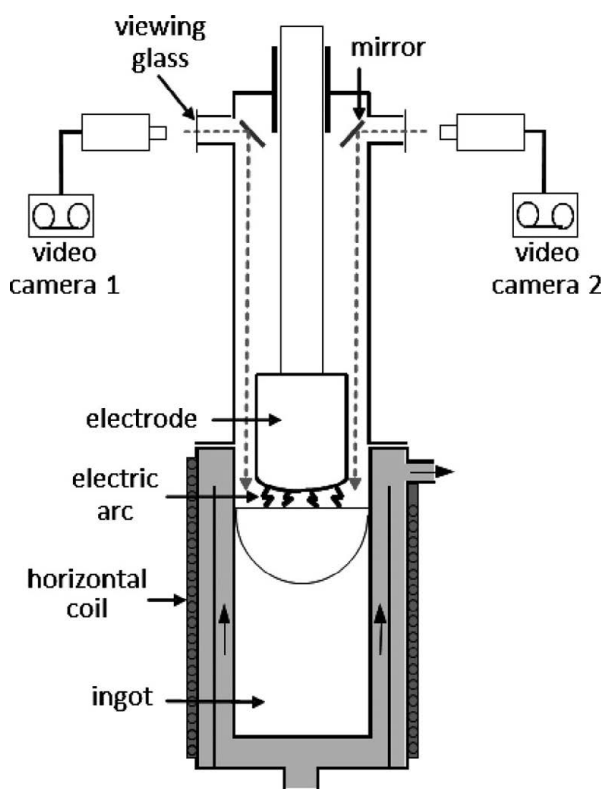

Fig. 5. VAR furnace equipped with two video cameras.

Table 1. Dominating periods of the luminosity fluctuations for different stirring sequences.

\begin{tabular}{lcc}
\hline \multicolumn{1}{c}{ Stirring sequence } & camera 1 & camera 2 \\
\hline \#1 No stirring & $28.1 \mathrm{~s}$ & $29.9 \mathrm{~s}$ \\
\#2 Unidirectional (very low B) & $29.9 \mathrm{~s}$ & $23.9 \mathrm{~s}$ \\
\#3 Alternated (low B, long period) & $28.1 \mathrm{~s}$ & $28.1 \mathrm{~s}$ \\
\#4 Alternated (high B, short period) & $43.5 \mathrm{~s}$ & $43.5 \mathrm{~s}$ \\
\#5 Alternated (low B, short period) & $28.1 \mathrm{~s}$ & $28.1 \mathrm{~s}$ \\
\hline
\end{tabular}

under usual practice to stir the metal in the liquid pool and to confine the arc underneath the electrode). These conditions differ by the type (unidirectional or alternating with a periodic reversal of the field direction) and the magnitude of the magnetic field. Note that visualisations were also performed in the absence of any magnetic field.

To help interpreting the recorded films, a specific image processing procedure similar to that proposed by Ward et al. ${ }^{11)}$ was developed. The main steps of this procedure are summarized below. First, the recorded film was split into a series of images. From these images, a new series of images was then generated by applying a $2 \mathrm{~s}$ moving average in order to suppress high frequency fluctuations related to individual cathode spot behaviour and by reducing the sampling frequency to 5 frames/s. In a third step, a given region of interest was extracted from each image and all the results were put side by side to build a temporal sequence (Fig. 6). Finally, the average luminosity in each extracted region was quantified and a Fourier analysis was performed to determine the frequencies of luminosity fluctuations along the temporal sequence.

An example of two temporal sequences corresponding to two diametrically opposite regions is illustrated in Fig. 7 for the case of stirring conditions \#3 (see Table 1). Each sequence spans a 4 min time interval. A plot of the temporal evolution of the average luminosity for both sequences is also presented on the figure.

The analysis of Fig. 7 revealed some unexpected behav- iour, which was not visible on the unprocessed images. The luminosity fluctuates quite regularly along both sequences, with an alternation between very bright time periods and other time periods during which the luminosity is notably reduced. The bright time periods are generally shorter than the darker ones. The fluctuations of luminosity in the two diametrically opposed regions are essentially in phase opposition, i.e. when the luminosity passes through a maximum in a region, it tends to be minimum in the opposite region. A frequency analysis indicates that the fluctuations involve several periods, with a dominating period of the order of 30 $\mathrm{s}$, which is identical for the two cameras.

These fluctuations may be related to the arc behaviour. Indeed, it can reasonably be considered that the luminosity fluctuates as a consequence of the evolution of the cathode spots forming the arc, due to their motion over the surface of the electrode. Namely, a high luminosity indicates the presence of an important number of spots on the electrode surface close to this region, whereas a low luminosity denotes the presence of a small number of spots (or even the absence of spots) nearby. On the basis of this assumption, the fluctuations of the luminosity described above indicate that the presence of the spots in a given region tends to vary periodically with time. Moreover, the fact that the fluctuations on both sides are not in phase reveals that the cathode spots are not evenly spread over the cathode surface and that the centre of gravity of their distribution (which can be regarded as the centre of gravity of the arc) is off-centre with respect to the axis of symmetry of the electrode. The combination of these two findings may be interpreted as an evidence of the existence of an apparent macroscopic motion of the centre of gravity of the arc across the electrode surface (with a period of about $30 \mathrm{~s}$ ). Namely, while the spots tend to cover more or less the whole electrode surface in a time average sense, their spreading behaviour may be such that the centre of their distribution tends to move macroscopically over the surface with a given period. The precise nature of this motion, which could be either a rotational or a translation displacement, cannot be determined from the present results.

Note that an alternative scenario could lead to similar luminosity fluctuations as those shown in Fig. 7. In this scenario, the centre of the arc would be off-centre but stationary, with spots arising at the centre location and expanding radially outward towards the electrode periphery where they extinguish. However, this latter scenario must be rejected, as the time necessary for the spots to spread over the electrode radius (of the order of several milliseconds, given a typical velocity of $10 \mathrm{~m} / \mathrm{s}^{15)}$ ) is much less than the time scale of the luminosity fluctuations measured in our study.

This phenomenon was observed most of the time for all the melt conditions tested. As shown in Table 1, the dominant period of the luminosity fluctuations is of the same order of magnitude (30 to $40 \mathrm{~s}$ ) for all melt conditions (including the conditions without any stirring). It seems in particular to be unconnected to the reversal period of the magnetic field. The reason for the larger value of the period in the case of high field conditions (melt conditions \#4) remains unclear at this stage. Thus, an ensemble motion of the arc seems to exist for various operating conditions and it appears to be relatively independent of the presence of an 
external axial magnetic field. Nevertheless, it should be mentioned that in the absence of an external magnetic field (melt conditions \#1), the fluctuations were less regular and the occurrence of relatively long periods with no significant fluctuations was more frequent. In contrast, the fluctuations of the luminosity were the most regular in the case of a strong magnetic field with a short alternating period (melt conditions \#4). A possible explanation for this could be a more efficient confinement of the cathode spots underneath the electrode due to a higher intensity of the magnetic field.

This experimental part of our work enables us to confirm the conclusions reached by Ward et al. ${ }^{12)}$ who reported a value of the time constant of the arc motion very similar as the one determined here. As discussed previously, the existence of a slow motion of the arc centre (with a time period of around $30 \mathrm{~s}$ ) could have important implications for the modelling of the VAR process as computational models based on a 2D axisymmetric geometry ${ }^{2,5)}$ might not be sufficient to describe the ingot and electrode behaviours (such models use integration time steps smaller than $1 \mathrm{~s}$ ).

The effects of a non-axisymmetric and time varying distribution of the arc on the electrode behaviour (in terms of variations of the shape of the electrode tip) are more partic-

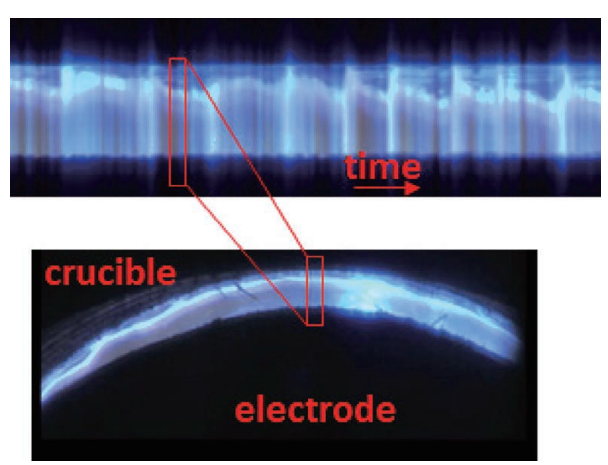

Fig. 6. Temporal sequence used to study the fluctuations of luminosity above the ingot top. ularly studied in the subsequent sections. The displaced centre of gravity of the electric arc is referred to as an 'arc-spot' in the rest of this paper. Several questions clearly arise, namely:

- Does an arc-spot motion induce a non-axisymmetry in the shape of the consumable electrode?

- What are the effects of various parameters of arc-spot like angular speed, power, distance from the centre, etc. on the cathode tip shape?

- What causes this arc-spot motion?

- Does the evolution of the cathode tip shape affects the arc-spot motion, i.e. is there any back effect?

These are some of the points which need to be answered for a better understanding of the VAR process. The aim of this paper is to give a clearer insight as far as the first two points are considered.
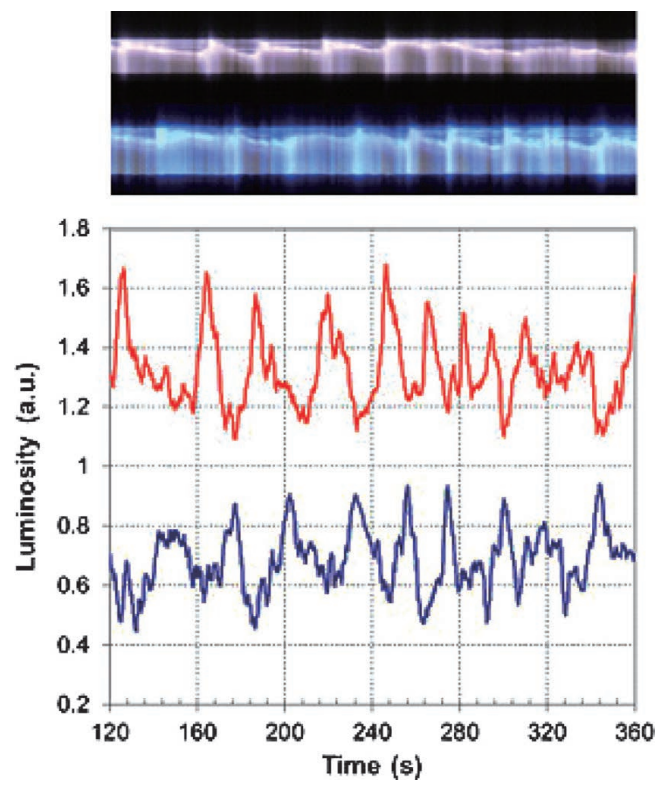

Fig. 7. Typical temporal sequences obtained for two diametrically opposite regions.

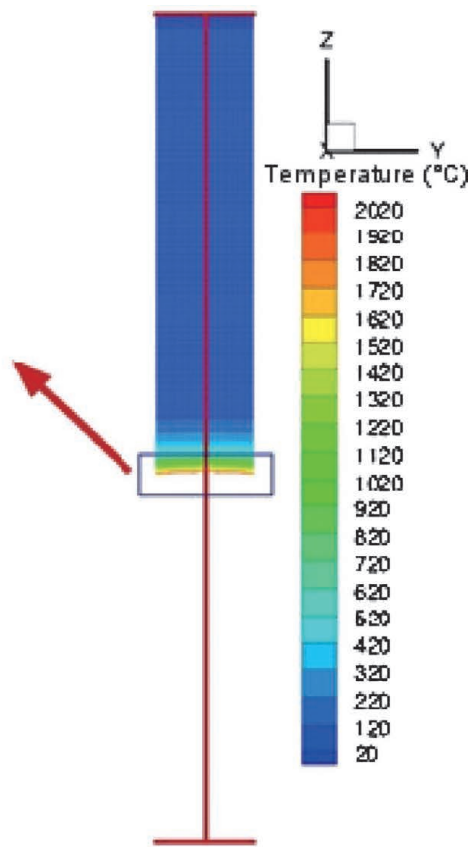

Fig. 8. Computed temperature field $\left({ }^{\circ} \mathrm{C}\right)$ in the cylindrical electrode $-\mathrm{Zy} 4$ remelting, uniform arc flux. 


\section{Modeling of the Cathode Melting}

This section briefly describes the numerical modelling of the transient thermal behaviour of the cathode. Basically, it is based on the 1D approach developed much earlier by Bertram and Zanner, ${ }^{16)}$ as well as Jardy et al.${ }^{17)}$ However, an original feature of the present model is the representation of liquid metal removal at the electrode base, which allows its use to predict the time evolution of the melt rate and the shape of the electrode tip for given operating parameters.

\subsection{Heat Transfer Equation}

Within the electrode, heat is transferred by conduction. Heating of the electrode by Joule effect is neglected, because of the very low resistivity of the metal to be remelted. Under these conditions, the heat transport equation is written in an enthalpic formulation: ${ }^{18)}$

$$
\frac{\partial}{\partial t}(\rho h)=\nabla \cdot(\lambda \nabla T)
$$

where $h$ is the mass average enthalpy, $T$ the temperature, $\rho$ the density and $\lambda$ the thermal conductivity. The temperature dependence of all alloy thermophysical properties is taken into account.

In Eq. (1), assuming a constant and equal specific heat in the solid and liquid phases, the enthalpy can be expressed as a function of the temperature and liquid fraction: $h=\mathrm{C}_{\mathrm{p}} \mathrm{T}+$ $\mathrm{g}_{1} \mathrm{H}_{\text {lat }}$, where $\mathrm{C}_{\mathrm{p}}$ is the specific heat, $\mathrm{g}_{1}$ the liquid fraction and $\mathrm{H}_{\text {lat }}$ the latent heat of melting. Thus, solving Eq. (1) requires knowledge of the solidification path, i.e. the evolution of the liquid fraction with temperature.

\subsection{Boundary Conditions}

To obtain a full definition, boundary and initial conditions must be specified. Initially, the electrode temperature is considered to be homogeneous and equal to the stub temperature. At the top of the electrode, a contact resistance between the electrode and stub is taken into account while at the electrode side, heat transfer is controlled by thermal radiation between the electrode and mould. The value of the emissivity is assumed to remain constant throughout the melting process.

At the electrode base, the power brought by the electric arc (originating from localised Joule heating due to the high current density or/and bombardment of the surface by the plasma ions) is expressed by a condition of the Neumann type (imposed flux density). The total energy flux density supplied by the electric arc must obey the relation:

$$
\int_{0}^{2 \pi} \int_{0}^{R_{\text {elec }}} \varphi(r, \theta, t) r d r d \theta=\chi P_{t o t}(t)
$$

where $R_{\text {elec }}$ is the electrode radius and $\chi$ is the fraction of the total power $P_{\text {tot }}(t)=\mathrm{U}_{\text {arc }} \mathrm{I}_{\text {arc }}$ that is transferred to the electrode. The distribution of the energy flux density brought by the arc at the electrode tip is an input parameter of the model. Indeed, Figs. 3 and 4 schematically present this distribution in two cases (2D and 3D versions).

The 3D model considers the existence of an arc-spot liable to move (as an example, a simple rotational motion is considered in Fig. 4) on the electrode base. This oversimplified representation enables us to account for an ensemble arc motion.

The total power transferred to the cathode $\chi P_{t o t}$ is the sum of a part distributed uniformly all over the bottom surface of the cathode and a part concentrated in the small area of the arc-spot. The boundary condition is then, at every moment:

$$
\varphi(r, \theta, t)=\frac{(1-\alpha) \chi P_{\text {tot }}(t)}{\pi R_{\text {elec }}^{2}}+\frac{\alpha \chi P_{\text {tot }}(t)}{\pi R_{\text {spot }}^{2}} f(r, \theta, t)
$$

and the second term on the r.h.s. of Eq. (3) is only applied if the considered location is concerned by the arc-spot ( $f=$ 1 under the arc-spot, and $f=0$ outside). The arc-spot focus $R_{\text {spot }}$, as well as the variable $\alpha$, are input parameters of the model. Obviously, setting $\alpha=0$ degenerates the 3D model into an axisymmetric one.

\subsection{Calculation Procedure}

The heat transfer equation is discretized using a finite volume method and fully implicit time scheme. ${ }^{19)}$ During each time step, we first calculate the temperature field in the electrode from Eq. (1). For each mesh cell that has no bottom neighbour, i.e. that is exposed to the arc, the energy flux received is simply calculated as:

$$
\phi_{b o t}(i)=\iint_{S(i)} \varphi(r, \theta, t) r d r d \theta
$$

where index i refers to the cell in the finite volume mesh and $S(i)$ is the area of the bottom surface of the cell. At each time step, the new position of the arc-spot is calculated, and $\phi_{b o t}(i)$ is determined according to Eqs. (3) and (4).

Then, in order to simulate the consumption of the electrode associated to the fall of liquid metal droplets formed at the electrode tip, mesh cells whose temperature is greater than an "overheating temperature" $T_{\text {liq }}+\Delta T_{\text {ov }}$ are removed from the computational domain. After cell removal, the boundary conditions are set at the new electrode tip for the next time step. Calculations are performed until fully consumption of the electrode.

It is important to notice that the melting of the electrode may induce some numerical irregularities in the shape of the base. The simple approach presented here can therefore be improved by a careful distribution of the arc energy in such regions. In the $2 \mathrm{D}$ case, it has been shown ${ }^{20)}$ that such a modification leads to only minor modifications in the cathode tip behaviour. However, this remains to be done in the 3D case.

Typical results of the model are the computed $\mathrm{T}(\mathrm{r}, \theta, \mathrm{z})$ temperature map at any moment in the remaining electrode, hence the instantaneous shape of the electrode base, as well as the evolution of the electrode weight and overall melting rate with time. An additional interesting feature of the model is its ability to account for the thermal effect of a crack located at the interface between two cells (e.g. the welding of electrode pieces) by means of a thermal resistance. ${ }^{21)}$

\subsection{Model Validation}

The 3D model has been used (with $\alpha=0$ ) to simulate the melting of a full-scale Zy4 electrode that had been presented previously ${ }^{22)}$ when only a $2 \mathrm{D}$ version of the model (implicitly setting $\alpha$ to 0 ) was available. The thermophysical prop- 
Table 2. Thermophysical properties of the cathode material (zirconium alloy Zy4).

\begin{tabular}{ll} 
Thermal conductivity $\lambda(\mathrm{W} / \mathrm{m} / \mathrm{K})$ & 43.5 \\
Density $\rho\left(\mathrm{kg} / \mathrm{m}^{3}\right)$ & $6.24 \times 10^{3}$ \\
Specific heat $\mathrm{C}_{\mathrm{p}}(\mathrm{J} / \mathrm{kg} / \mathrm{K})$ & $4.61 \times 10^{2}$ \\
Latent heat of melting $\mathrm{H}_{\text {lat }}(\mathrm{J} / \mathrm{kg})$ & $2.19 \times 10^{5}$ \\
Emissivity $\varepsilon$ & 0.33 \\
Phase change temperatures $\mathrm{T}_{\text {sol }}-\mathrm{T}_{\text {liq }}\left({ }^{\circ} \mathrm{C}\right)$ & $1800-1830$ \\
\hline
\end{tabular}

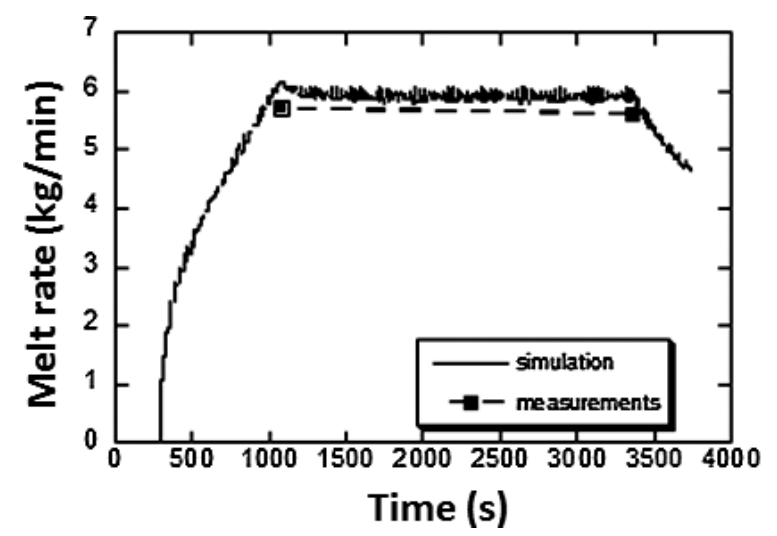

Fig. 9. Time evolution of both monitored and calculated melt rates Zy4 remelting.

erties of the cathode material are listed in Table 2.

The 2D model predicted that the most important temperature axial gradient remained confined at the bottom, close to the electrode tip. Also, the concave shape of the base resulted directly from the thermal losses by radiation along the lateral surface of the electrode. All these conclusions are exactly confirmed when the 3D model is used as the situation degenerates to axisymmetry when no ensemble arc motion is accounted for, so numerical results are identical.

Figure 8 shows the computed temperature field of the cathode at around half of the overall melting duration. The insert zoom enables us to visualize the concave shape of the electrode base.

Figure 9 depicts the temporal evolution of the predicted melt rate of the electrode as well as the melt rate monitored during the time interval throughout which the arc power was maximum. The variation in the overall melt rate, including an initial pre-heating stage followed by a sharp increase of the melt rate until reaching a quasi-stationary regime, is well described by the model. The average calculated melt rate was constant (equal to $5.9 \mathrm{~kg} / \mathrm{min}$ ) throughout most of the melt. This value was found to be in excellent agreement with that monitored during the real melt $(5.7 \mathrm{~kg} / \mathrm{min})$, which validates the model.

\section{Influence of an Ensemble Arc Motion: Results and Discussion}

In this section, we study the influence of a non-zero value of the parameter $\alpha$, which implies that part of the heat input at the cathode tip is not uniformly distributed, but rather through an arc-spot moving on the electrode base. The main operating parameters for all calculations discussed below are reported in Table 3. Such operating parameters do not
Table 3. Operating parameters used for all simulations.

\begin{tabular}{ll}
\hline Electrode radius $\mathrm{R}_{\text {elec }}(\mathrm{m})$ & 0.1 \\
Melting current $\mathrm{I}_{\text {arc }}(\mathrm{A})$ & $6.5 \times 10^{3}$ \\
Arc voltage $\mathrm{U}_{\mathrm{arc}}(\mathrm{V})$ & 32 \\
Fraction of power transmitted to the cathode $\chi$ & 0.58 \\
Overheating temperature interval $\Delta \mathrm{T}_{\mathrm{ov}}\left({ }^{\circ} \mathrm{C}\right)$ & 100 \\
\hline
\end{tabular}

Table 4. Input data for the whole set of runs.

\begin{tabular}{ccccc}
\hline Serial $\mathrm{n}^{\circ}$ & $\begin{array}{c}\text { Arc-spot } \\
\text { power } \\
\text { fraction } \alpha\end{array}$ & $\begin{array}{c}\text { Arc-spot } \\
\text { focus } \\
R_{\text {spot }}(\mathrm{m})\end{array}$ & $\begin{array}{c}\text { Radial } \\
\text { location } \\
\text { dist }(\mathrm{m})\end{array}$ & $\begin{array}{c}\text { Motion } \\
\text { frequency } \\
f(\mathrm{rpm})\end{array}$ \\
\hline $\begin{array}{c}\text { (uniform } \\
\text { density) }\end{array}$ & 0 & $/$ & $/$ & $/$ \\
2 (reference) & $\mathbf{0 . 0 5}$ & $0.2 R_{\text {elec }}$ & $\underline{0.7 \mathrm{R}_{\text {elec }}}$ & 2 \\
3 & $\mathbf{0 . 0 2 5}$ & $0.2 \mathrm{R}_{\text {elec }}$ & $0.7 \mathrm{R}_{\text {elec }}$ & 2 \\
4 & $\mathbf{0 . 1}$ & $0.2 \mathrm{R}_{\text {elec }}$ & $0.7 \mathrm{R}_{\text {elec }}$ & 2 \\
5 & 0.05 & $0.1 R_{\text {elec }}$ & $0.7 \mathrm{R}_{\text {elec }}$ & 2 \\
6 & 0.05 & $0.3 R_{\text {elec }}$ & $0.7 \mathrm{R}_{\text {elec }}$ & 2 \\
7 & 0.05 & $0.2 \mathrm{R}_{\text {elec }}$ & $\underline{0.3 \mathrm{R}_{\text {elec }}}$ & 2 \\
8 & 0.05 & $0.2 \mathrm{R}_{\text {elec }}$ & $\underline{0.5 \mathrm{R}_{\text {elec }}}$ & 2 \\
9 & 0.05 & $0.2 \mathrm{R}_{\text {elec }}$ & $\underline{\text { spiral }}$ & 2 \\
10 & 0.05 & $0.2 \mathrm{R}_{\text {elec }}$ & $0.7 \mathrm{R}_{\text {elec }}$ & $\mathbf{1}$ \\
11 & 0.05 & $0.2 \mathrm{R}_{\text {elec }}$ & $0.7 \mathrm{R}_{\text {elec }}$ & 4 \\
12 & 0.05 & $0.2 \mathrm{R}_{\text {elec }}$ & $0.7 \mathrm{R}_{\text {elec }}$ & $\boldsymbol{8}$ \\
\hline
\end{tabular}

correspond to any actual melt. All runs were performed using an initial 3D meshing of 2700000 cells (100-90-300 in $\mathrm{r}-\theta-\mathrm{z}$ directions).

As compared to the concave and almost flat electrode generally obtained in the case of uniform heating, ${ }^{21,22)}$ the presence of an arc-spot results in a 3D shape of the electrode tip, even when a low value is set for $\alpha$. As an example, Fig. 10 shows the computed map of the electrode temperature at a given moment (the observation plane corresponds to the instantaneous arc-spot location).

In that simulation, the arc-spot is assumed to carry $\alpha=$ $5 \%$ of the total heat input. It rotates around the electrode centreline with a $30 \mathrm{~s}$ period. Its radius $R_{\text {spot }}$ is $20 \%$ of the electrode radius $R_{\text {elec }}$ and the location of its centre is dist $=$ $0.3 \mathrm{R}_{\text {elec. }}$ Such conditions correspond roughly to magnetic measurements by Ward et al. ${ }^{12)}$ The computed non-flatness of the electrode tip seems very pronounced in this case. Indeed, if a "concavity" is defined as $\left(\mathrm{H}_{\max }-\mathrm{H}_{\min }\right) / \mathrm{R}_{\text {elec}}$, where $H_{\max }$ (resp. $H_{\text {min }}$ ) is the maximum (resp. minimum) electrode height, its value reaches around 0.15 . This result does not agree with usual industrial observation of the cathode tip.

In order to study the influence of the arc behaviour on the melting process, a set of 12 runs has been achieved with various values for the main arc-spot parameters, that are:

- $\alpha$, the fraction of total power carried by the arc-spot

- $\mathrm{R}_{\text {spot, the arc-spot focus }}$

- dist, the radial location of the arc-spot centre

- $\mathrm{f}$, the frequency of its rotational motion

Table 4 details the values that have been used for the whole set of runs. Only the most significant results are 


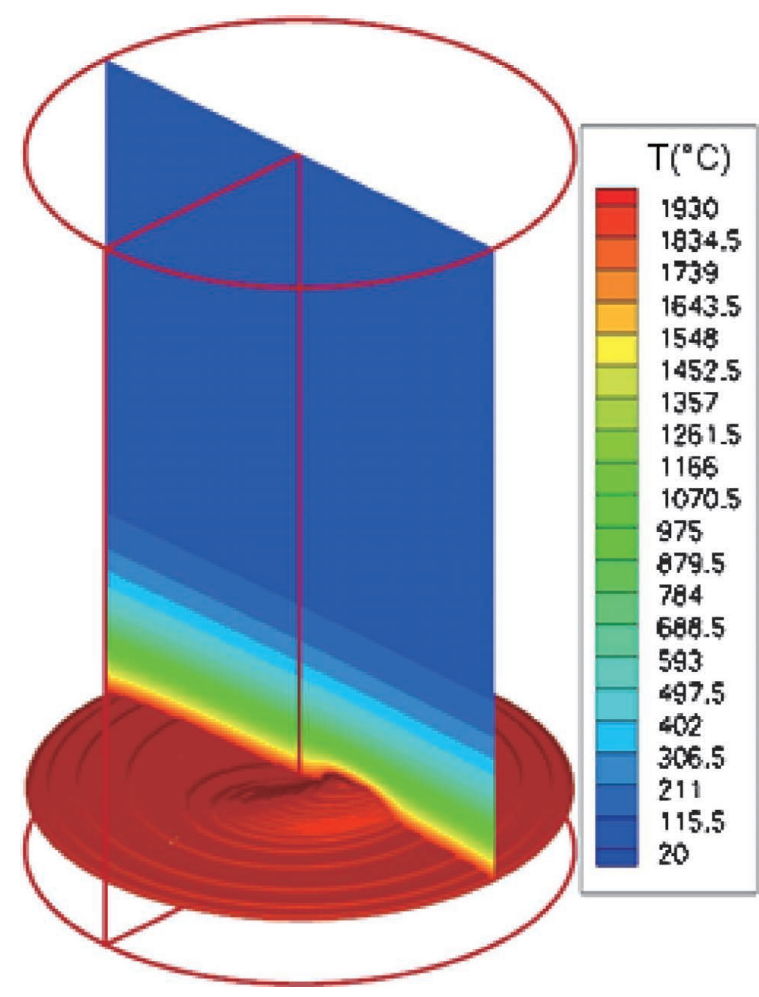

Fig. 10. Example of electrode temperature map, showing the influence of arc-spot motion.

reported below.

As we can see on Fig. 11, the radial location of the arcspot has a pronounced effect on the computed shape of the electrode tip.

The influence of the radial location of the arc-spot on the shape of the electrode tip can be explained by the fact that the time during which a given point is heated by the moving spot decreases proportionally to the inverse of its radial location. Thus for a given angular speed, the farther a point from the centre, the lower the time during which it will be heated by the spot and by the way the lower the deformation of the electrode tip. Indeed, only a rotating spot located close to the edge will not induce a non-planar and very irregular shape. Therefore, the value dist $=0.7 \mathrm{R}_{\text {elec }}$ was chosen to study the influence of $\alpha$. Not surprisingly, Fig. 12 clearly indicates that a very low value (less than 5\%) is required if the tip is supposed to remain relatively flat.

If the arc-spot carries only a small fraction of the overall arc energy, it is interesting to check if the measured luminosity fluctuations can be qualitatively confirmed by the model. To this end, we have defined a numerical "luminosity" in a very crude way, as:

$$
L=\sum_{i} \frac{\phi_{b o t}(i)}{d(i, O)}
$$

where $O$ is a fixed location on the cathode bottom edge $(\mathrm{r}=$ $\mathrm{R}_{\text {elec}}, \theta=0$ ) vertically facing a virtual video camera. Figure $\mathbf{1 3}$ gives an example of the variation of this computed 'luminosity' with time.

Any change in $\mathrm{R}_{\text {spot }}$ has no influence, as the three curves are almost identical. The periodic (30 s) variation is caused by the arc-spot rotation. Such a qualitative result is systematically obtained, as soon as $\alpha$ differs from 0 . In all cases, it is interesting to notice that high-luminosity periods (when

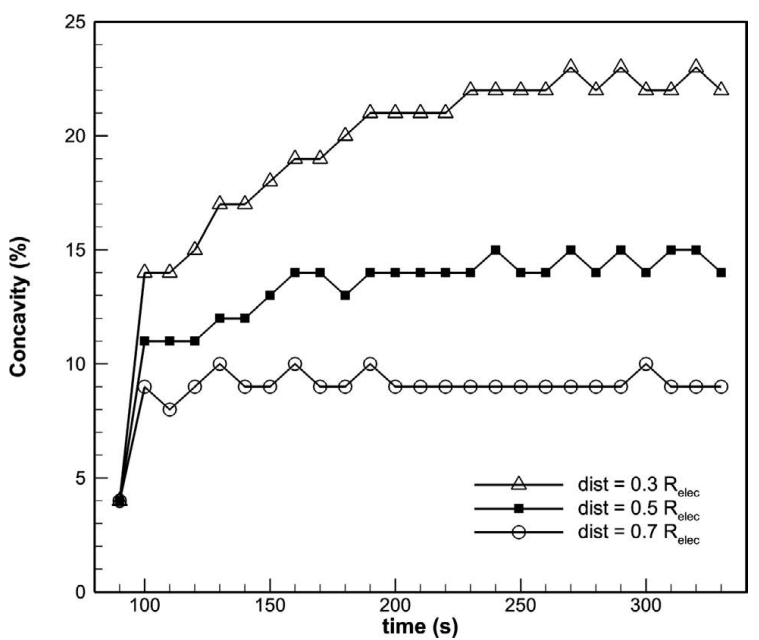

Fig. 11. Influence of the spot centre location on the "concavity" $(\alpha=$ $\left.0.05, \mathrm{R}_{\text {spot }} / \mathrm{R}_{\text {elec }}=0.2, \mathrm{f}=2 \mathrm{rpm}\right)$.

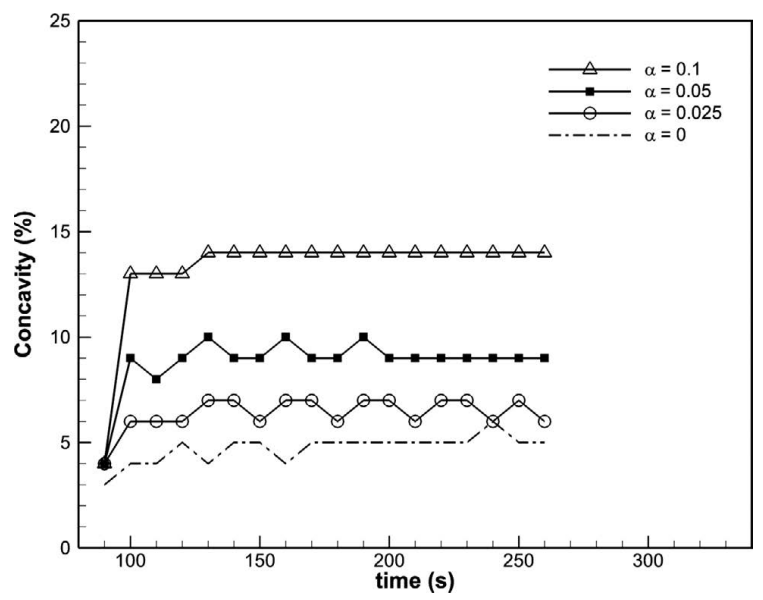

Fig. 12. Influence of $\alpha$ on the "concavity" (dist $/ \mathrm{R}_{\text {elec }}=0.7, \mathrm{R}_{\text {spot }} /$ $\left.\mathrm{R}_{\text {elec }}=0.2, \mathrm{f}=2 \mathrm{rpm}\right)$.

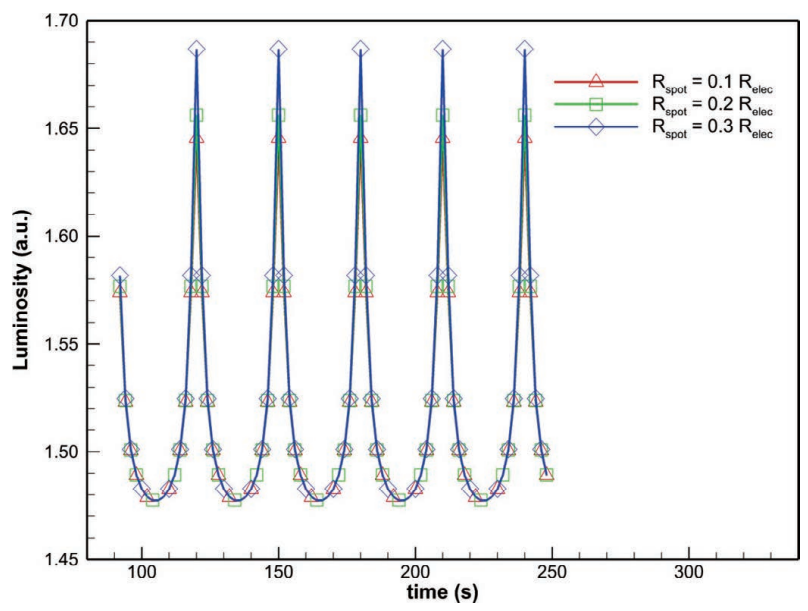

Fig. 13. Computed fluctuation of "luminosity" $\left(\alpha=0.05\right.$, dist $/ \mathrm{R}_{\mathrm{elec}}=$ $0.7, \mathrm{f}=2 \mathrm{rpm})$.

the arc-spot location approaches $O$ ) are shorter than lowluminosity ones. The comparison of Figs. 7 and 13 indicates that the motion of an arc-spot carrying only a small percentage of the arc power explains qualitatively the experimental observation. 


\section{Conclusions}

An extensive study of the behaviour of the electric arc in the VAR process has been undertaken at both micro- and macroscale. At the microscale, the arc appears as a collection of spot clusters moving at the cathode tip. Its behaviour corresponds to the diffuse operating mode. At the macroscale, observation of the arc luminosity fluctuations confirms experimental findings by Ward et al. ${ }^{11)}$

The development of a 3D model of the heat transfer in the consumable electrode and associated melting process enables us to determine some effects of the electric arc behaviour. In industrial practice, the cathode tip remains relatively flat during melting. Yet, an arc which consists in a uniform part and an arc-spot rotating around the axis of the electrode with a constant small radial position results in a concentration of part of the melting power in a given area. This would lead to a preferential melting of this part of the electrode, hence a non-flat electrode tip, in contradiction with the observation, unless the fraction of total power carried by the arc-spot remains very low (only a few\%). In the latter case, both luminosity periodic fluctuations and electrode flatness can be obtained.

Other possible ensemble arc motions can be investigated using the model. Apart from the rotational motion discussed above, a spiral-like motion (\#9 in Table 4) results in an electrode tip shape that remains flat enough, while still compatible with the periodic behaviour of the luminosity.

In all cases, the reasons for such a macroscopic motion of the arc are to be understood yet. The most difficult to interpret and striking feature remains the rather large time constant of this motion which is much greater than the time scales characteristic of the behaviour of individual cathode spots (in the range of $1 \mu \mathrm{s}$ to $1 \mathrm{~ms}$ ) and does not correspond either to any obvious time scales of the VAR process. Further experiments coupling the optical diagnostics used in the present study with direct visual observations of the arc appearance at the through side viewing ports, as used in some previous studies ${ }^{9,10)}$ could help to provide more conclusive data.

\section{REFERENCES}

1) T. Quatravaux, S. Ryberon, S. Hans, A. Jardy, B. Lusson, P. E. Richy and D. Ablitzer: J. Mater. Sci., 39 (2004), 7183.

2) A. Jardy and D. Ablitzer: Mater. Sci. Technol., 25 (2009), 163.

3) L. Falk, A. Jardy, D. Ablitzer and P. Paillere: Rev. Métall., 87 (1990), 209.

4) A. Jardy and D. Ablitzer: Rev. Métall., 87 (1990), 421.

5) A. Jardy and D. Ablitzer: Rare Metal Mat. Eng., 35 (2006), 119.

6) P. Chapelle, A. Jardy, J. P. Bellot and M. Minvielle: J. Mater. Sci., 43 (2008), 5734

7) F. J. Zanner: Metall. Trans. B, 10B (1979), 133

8) R. L. Williamson and F. J. Zanner: Proc. of 1991 Vacuum Metallurgy Conf. on Melting and Processing of Speciality Materials, AVS, New York, NY, (1992), 87.

9) P. Chapelle, J. P. Bellot, A. Jardy, T. Czerwiec, X. Robbe, B. Champin and D. Ablitzer: High Temp. Mater. Process, 4 (2000), 493.

10) P. Chapelle, T. Czerwiec, J. P. Bellot, A. Jardy, D. Lasalmonie, J. Senevat and D. Ablitzer: Plasma Source Sci. Tech., 11 (2002), 301.

11) R. M. Ward and M. H. Jacobs: J. Mater. Sci., 39 (2004), 7135.

12) R. M. Ward, B. Daniel and R. J. Siddall: Proc. of Int. Symp. on Liquid Metal Processing and Casting, ASM International, Materials Park, OH, (2005), 49.

13) L. Yuan, G. Djambazov, P. D. Lee and K. Pericleous: Int. J. Mod. Phys. B, 23 (2009), 1584.

14) V. Bojarevics, K. Pericleous, G. Djambazov and R. M. Ward: Proc. of Int. Symp. on Liquid Metal Processing and Casting, TMS, Warrendale, PA, (2009), 21.

15) R. L. Boxman, P. J. Martin and D. M. Sanders: Handbook of Vacuum Arc Science and Technology, Noyes Publications, Park Ridge, NJ, (1995), 257.

16) L. A. Bertram and F. J. Zanner: Proc. of Int. Conf. Modeling and Control of Casting and Welding Processes, TMS, Warrendale, PA, (1986), 95.

17) L. Falk, A. Jardy and D. Ablitzer: Ironmaking Steelmaking, 19 (1992), 226.

18) V. de Felice, A. Jardy and H. Combeau: Proc. of Int. Symp. on Liquid Metal Processing and Casting, TMS, Warrendale, PA, (2009), 97.

19) S. V. Patankar: Numerical Heat Transfer and Fluid Flow, Hemisphere Pub. Corp., New York, (1980), 58.

20) H. El Mir: PhD Thesis, Institut National Polytechnique de Lorraine, Nancy, France, (2005), 108.

21) H. El Mir, A. Jardy, J. P. Bellot, P. Chapelle, D. Lasalmonie and J. Senevat: J. Mater. Process. Technol., 210 (2010), 564.

22) J. P. Bellot, P. Zabeti, H. El Mir, J. Jourdan, P. Chapelle and A. Jardy: Proc. of Int. Symp. on Liquid Metal Processing and Casting, TMS, Warrendale, PA, (2009), 3. 